OPEN ACCESS

Edited by: Aamir Ahmad,

University of Alabama at Birmingham,

United States

Reviewed by:

Y-h. Taguchi,

Chuo University, Japan

Alex C. Kornke

University of York, United Kingdom

${ }^{*}$ Correspondence:

Jie Yang

yj197912@163.com

Zengjun Wang

zengjunwang@njmu.edu.cn

${ }^{t}$ These authors have contributed equally to this work

Specialty section: This article was submitted to Molecular and Cellular Oncology, a section of the journal

Frontiers in Oncology

Received: 29 September 2020 Accepted: 17 March 2021

Published: 14 April 2021

Citation:

Xue J, Zhu S, Qi F, Zhu K, Cao P, Yang $J$ and Wang $Z$ (2021) RUNX1/ miR-582-5p Pathway Regulates the Tumor Progression in Clear Cell Renal Cell Carcinoma by Targeting COL5A1.

Front. Oncol. 11:610992. doi: 10.3389/fonc.2021.610992

\section{RUNX1/miR-582-5p Pathway Regulates the Tumor Progression in Clear Cell Renal Cell Carcinoma by Targeting COL5A1}

\author{
Jianxin Xue ${ }^{1,2 \dagger}$, Shenhao $\mathrm{Zhu}^{2 \dagger}$, Feng $\mathrm{Qi}^{3 \dagger}{ }^{2}$, Kai Zhu ${ }^{1 \dagger}$, Pu Cao ${ }^{1}$, Jie Yang ${ }^{2 *}$ \\ and Zengjun Wang ${ }^{2 *}$ \\ 1 Department of Urology, The Second Hospital of Nanjing, Nanjing University of Chinese Medicine, Nanjing, China, \\ ${ }^{2}$ Department of Urology, The First Affiliated Hospital of Nanjing Medical University, Nanjing, China, ${ }^{3}$ Department of Urology, \\ Jiangsu Cancer Hospital, Nanjing Medical University, Nanjing, China
}

Recent evidences indicated that miRNAs played core role in the progression of clear cell renal cell carcinoma (ccRCC). However, its molecular mechanism in ccRCC is still remained unclear. The study was designed to identify the role and regulatory mechanism of miR-582-5p in ccRCC. In this study, the low expression level of miR$582-5 p$ were detected by qRT-PCR in ccRCC patient tumor samples and ccRCC cell lines, respectively. The expression level of miR-582-5p was associated with tumor stage and metastasis. In vivo and in vitro experiments found miR-582-5p inhibit tumor growth via suppressing COL5A1 expression. Additionally, RUNX1 was identified as the negative regulator of miR-582-5p through database prediction and chromatin immunoprecipitation. Finally, the negative relation of RUNX1 and miR-582-5p was verified through rescue experiment both in vitro and in vivo. In summary, miR-582-5p, which was regulated by RUNX1, inhibited tumor growth and invasion by targeting COL5A1, indicating that miR-582-5p may act as a biomarker and that the RUNX1/miR$582-5 \mathrm{p} / \mathrm{COL} 5 \mathrm{~A} 1$ axis could be a potential therapeutic target for ccRCC.

Keywords: ccRCC, miR-582-5p, Runx1, proliferation, invasion

\section{INTRODUCTION}

Renal cell carcinoma (RCC) is one of the most common and lethal malignancy with a global estimate for 403,262 new cases and 175,098 deaths according to the American Cancer Society (1). RCC contains three major histological subtypes clear cell RCC (ccRCC), papillary RCC (pRCC) and chromophobe RCC (chRCC) (2). ccRCC is the most common one which accounts for 75 percent among all RCC cases (3). Over the years, the incidence of RCC is gradually increasing probably due to an increase in the incidental detection of renal masses. In addition, although most of new diagnosed RCC tumor are localized, there are considerable cases with locally advanced diseases or metastases $(4,5)$. Hence, exploring the accurate factors and mechanisms responsible for tumorigenesis or development is the main goal, which may be valuable for early diagnosis and outcome predicting (6). 
MicroRNAs are single-stranded non-coding RNAs containing approximately 23 nucleotides. They function as regulator of gene expression through binding to the 3' untranslated region (3'UTR) of selected messenger RNA (mRNA). As a consequence, they induce either degradation or translational repression of mRNAs $(7,8)$. Previous evidences demonstrated that miRNAs are involved in several biological processes, such as cell growth, apoptosis, differentiation, and tumor tumorigenesis (9-12). As a member of miRNAs family, numerous studies showed that miR-582-5p was critical to the development of several solid tumors, including glioblastoma, prostate cancer, gastric cancer, lung cancer, oral cancer, colorectal cancer, bladder cancer, salivary adenoid cystic carcinoma, hepatocellular carcinoma, etc. (13-23). Unexpectedly, although a majority of studies verified that miR-582-5p is a tumor suppressor, other studies held different opinions. For instance, Maeno A et al. proved that miR-582-5p contributed to an increase in the proliferation of prostate cancer cells under androgen deprived conditions (20). Similar conclusion was draw by Shu Z et al. that miR-582-5p induced progression of colorectal cancer (17). Considering these controversial results, we were interested in the role of miR-582-5p in RCC, which still remained unclear.

In the present study, we showed that miR-582-5p was downregulated in ccRCC cell lines and tissues, and over-expression of miR-582-5p suppressed the growth and migration in vitro and in vivo. Interestingly, our findings indicated that miR-582-5p, which was mediated by RUNX1, accelerated ccRCC progression through promoting the expression of COL5A1.

\section{MATERIALS AND METHODS}

\section{Tissue Specimens}

From April to August 2019, 40 pairs of ccRCC tissues and their adjacent normal tissues were collected from patients following radical nephrectomy at Jiangsu Province Hospital, First Affiliated Hospital of Nanjing Medical University. The project was approved by the Ethics Committee of author's institution. All procedures on the enrolled patients were informed in advance with written consent. All fresh samples were gathered after surgery and frozen in liquid nitrogen immediately and then stored at $-80^{\circ} \mathrm{C}$ refrigerator until RNA extraction.

\section{Cell Culture and Transfection}

Human renal proximal tubule epithelial cell line HK-2 and human ccRCC cell lines 786-O and Caki-1 were purchased from the Chinese Academy of Sciences (Shanghai, China). HK2 cells were cultured in DMEM-F12k medium (Thermo Fisher Scientific, Waltham, MA, USA), and other cells were cultured in RPMI-1640 medium (Thermo Fisher Scientific) supplemented with 10\% FBS (TransGen Biotech, Beijing, China), $100 \mathrm{U} / \mathrm{mL}$ penicillin, and $100 \mu \mathrm{g} / \mathrm{mL}$ streptomycin (Solarbio, Beijing, China). Cells were maintained in a humidified incubator at $37^{\circ} \mathrm{C}$ in $5 \% \mathrm{CO} 2$.

Besides, selected cell lines were transfected with pcDNA3.1COL5A1 and pcDNA3.1-RUNX1 (GenePharma, Shanghai, China). The mimics for miR-582-5p was also obtained from GenePharma. Transfection of these into cells was performed with Lipofectamine 2000 (Invitogen; Thermo Fisher Scientific, Waltham, MA, USA) following the manufacturer's protocol.

\section{RNA Extraction and Quantitative Real-Time PCR (qRT-PCR)}

Total RNA was isolated from tissues and cells using TRIzol Reagent (Invitrogen, CA, USA). cDNA was synthesized from $2 \mu \mathrm{g}$ of total RNA using a SuperScript II Reverse Transcriptase kit (Invitrogen). qRT-PCR was performed using an the Power SYBR Green Master Mix (Applied Biosystems). The comparative threshold cycle (CT) $\left(2^{-\Delta \Delta C t}\right)$ method was used to analysis the relative fold changes in gene expression. The U6 and GAPDH were used as internal controls.

\section{Protein Isolation and Western Blot Assay}

Proteins were extracted from the cultured cells with RIPA lysis buffer (1\% NP40, 0.1\% sodium dodecyl sulfate (SDS), $100 \mu \mathrm{g} / \mathrm{ml}$ phenylmethyl sulfonyl fluoride, and $0.5 \%$ sodium deoxycholate, in PBS) on ice. The supernatants were collected after centrifugation at $12000 \times \mathrm{g}$ at $4^{\circ} \mathrm{C}$ for $20 \mathrm{~min}$. After the protein concentration was determined using a BCA protein assay kit (Bio-Rad, China), the lysates were mixed with $4 \times$ SDS loading buffer ( $125 \mathrm{mmol} / \mathrm{l}$ Tris$\mathrm{HCl}, 4 \%$ SDS, $20 \%$ glycerol, $100 \mathrm{mmol} / \mathrm{l} \mathrm{DTT}$, and $0.2 \%$ bromophenol blue) at a ratio of 1:3. After the samples were heated at $100^{\circ} \mathrm{C}$ for 5 min proteins were differentiated on SDS polyacrylamide gels. The separated proteins were then transferred to a PVDF membrane. The membrane blots were probed with a primary antibody of rabbit IgG anti-COL5A1 $(1: 1,000)$ or rabbit IgG anti-glyceraldehyde 3-phosphate dehydrogenase $(1: 1,000)$ (Cell Signaling Technology Inc., Boston, MA, USA) and horseradish peroxidase-conjugated anti-rabbit IgG (Jackson ImmunoResearch Labs, West Grove, PA, USA) secondary antibody, and developed with the enhanced chemiluminescent system. The signals were recorded using X-ray film. GAPDH was used as an internal reference for protein COL5A1 and RUNX1.

\section{Cell Proliferation Assay}

The proliferation ability of cells was evaluated using the Cell Counting Kit-8 (CCK-8, Dojindo, Kumamoto, Japan) after transfection. Twenty-four hours after transfection, cells were planted into 96-well plates (3x103 cells/well). For concentration gradient assay, $10 \mu \mathrm{L}$ of CCK- 8 reagent was added into each well of the 96-well plates after incubation for $48 \mathrm{~h}$, while being added at various time points $(24 \mathrm{~h}, 48 \mathrm{~h}, 72 \mathrm{~h}$ and $96 \mathrm{~h})$ for time gradient assay. Then the 96 -well plates were placed at $37^{\circ} \mathrm{C}$ for $2 \mathrm{~h}$, and the absorbance at $450 \mathrm{~nm}$ was measured using Infinite $\mathrm{F} 50^{\circledR}$ microplate reader (Tecan, $\mathrm{CH}$ ), Subsequently. Each experiment was carried out in four-replicate wells and repeated three times.

\section{Colony Formation Assay}

48 hours after cell transfection, 786-O or Caki- 1 cells $\left(1 \times 10^{3}\right.$ cells per well) was seeded into a six-well plate for 14 days. After fixed with $10 \%$ formaldehyde for $20 \mathrm{~min}$, the forming colonies were then stained with $0.1 \%$ crystal violet (Sigma-Aldrich Co.). Then the number of foci $>100$ cells was counted. 


\section{Cell Invasion Assay}

Transwell assay was carried out to explore the invasive and migratory capacities of ccRCC cells with $8-\mu \mathrm{m}$ pore sized transwell chamber (BD Biosciences, San Jose, CA, USA). For invasion assay, the upper chambers were precoated with a matrigel (BD Biosciences). The transfected cells at $5 \times 10^{3}$ cells/ well resuspended in FBS-free medium were plated into the top chamber. Medium containing $10 \%$ FBS was added to the bottom chambers. After incubated for $48 \mathrm{hrs}$, the noninvading or migrating cells were scraped whereas cells adhering to the bottom surface of the upper chamber were fixed and stained. Finally, the numbers of cells were calculated by counting five random fields under the microscope (Olympus). All assays were performed in triplicate.

\section{Xenograft Model}

Murine xenografts were established in 6- to 8-week-old male nude mice (BALB/c-null) using 786-O or Caki-1 cells. A total volume of $150 \mu \mathrm{l}$ containing $2.5 \times 106$ tumor cells were inoculated subcutaneously into the backs. Each group consisted of 5 animals. Tumor growth was monitored every 4 days in each group. Tumor volume was calculated (width ${ }^{2} \times$ length) $/ 2$ at each time point. The mice were sacrificed 28 days later, and the tumors were photographed and stained with hematoxylin/eosin. All animal studies were approved by the animal ethics committee of Nanjing Medical University.

\section{Immunohistochemistry (IHC)}

All the extracted samples were fixed with formalin (4\%) and embedded in paraffin. Mice tumor tissues were sliced into tissue sections ( $5 \mu \mathrm{m}$ thickness) and incubated with antibodies for Ki-67 (Abcam, ab92742) overnight at $4{ }^{\circ} \mathrm{C}$. The sections were incubated with the secondary HRP Goat Anti-Rabbit IgG (Abcam, ab205718) at room temperature for $1 \mathrm{~h}$, and then were stained with DAB solution. The nuclei were counterstained with hematoxylin. An Olympus microscope (Olympus, Tokyo, Japan) was used to evaluate the images.

\section{Mammalian Lentiviral Overexpression Plasmid Construction}

The coding sequence of COL5A1 and RUNX1 was amplified and cloned into pLEX-MCS, and the pLEX-GFP was used as a control. To generate lentiviral particles, plasmids were co-transfected into HEK293T cells along with envelope (VSVG) and packaging (Delta 8.9) plasmids using Lipofectamine 2000 (Invitrogen). The viral supernatants were harvested and filtered after two days of transfection. Cells were infected with $8 \mu \mathrm{g} / \mathrm{ml}$ polybrene. Overexpression efficiencies were confirmed via western blotting.

\section{Bioinformatics Database Analysis}

An online tool dbDEMC 2.0 (https://www.picb.ac.cn/dbDEMC/ index.html) was used to obtain the general information of miR582-5p. Publicly available tools including TargetScan (http:// www.targetscan.org/), miRanda (http://www.microrna.org/), miRDB (http://www.mirdb.org/) and mirWalk (http://zmf. umm.uni-heidelberg.de/apps/zmf/mirwalk2/) were used to predict the candidate target genes of miR-582-5p. And also TransmiR (http://www.cuilab.cn/transmir) was used to identify upstream regulators that may be responsible for miR-582-5p.

\section{Dual-Luciferase Reporter Assay}

The mutant (MUT) or wild-type (WT) sequences containing the predicted target site of miR-582-5p in the 3'UTRs of COL5A1 mRNA were inserted into the mir-GLO vectors (Promega Corporation, Madison, WI, USA). For the luciferase reporter assay, $786-\mathrm{O}$ and Caki- 1 cells $\left(1 \times 10^{5}\right.$ cells per well) were seeded in 24-well plates and co-transfected with $300 \mathrm{ng}$ of WT or MUT luciferase vector together with30nM of either miR-582-5p mimics or miR-NC. Then the luciferase activity was determined at $48 \mathrm{~h}$ after transfection using a Dual-Luciferase ${ }^{\circledR}$ Assay System (Promega) according to the manufacturer's instructions.

\section{Chromatin Immunoprecipitation (ChIP)}

ChIP analysis was conducted using ChIP assay kit (Millipore, 17610 ) based on the manufacturer's instructions. A total of $1 \times 10^{7}-$ $5 \times 10^{7}$ cells were collected and $1 \%$ formaldehyde (Bio-Rad, CA, USA) was used to crosslink the proteins to the DNA for $25 \mathrm{~min}$. Chromatin was sheared to fragments with size of 100-500 bp by sonicating the lysate. After dislodged insoluble substance by centrifugation, $100 \mu \mathrm{l} \mathrm{DNA}$ /protein complexes were taken as input. The samples were incubated with RUNX1 antibody (Cell Signaling Technology), normal rabbit IgG antibodies (Cell Signaling Technology), and protein $\mathrm{A} / \mathrm{G}$ beads overnight at $4^{\circ} \mathrm{C}$. After incubation at $65{ }^{\circ} \mathrm{C}$ for $4 \mathrm{~h}$, the crosslinking of input and the samples were reversed. Then, phenol/chloroform (Invitrogen) was involved to recover DNA from the samples. Promoter binding was evaluated via PCR with primers of miR-582-5p upstream region.

\section{Statistical Analysis}

All of the experiments were performed independently three times. Results were described as means \pm standard deviation (SD). All statistical analysis was performed using GraphPad Prism software version 6.0 (GraphPad, Software, Inc., La Jolla, CA, USA). The correlation between miR-582-5p and COL5A1 expression in RCC tissues was determined using Pearson's correlation coefficient by GraphPad Prism 6.0 software. Statistical differences were evaluated using Student's t-test for two-group comparisons and one-way analysis of variance for more than two groups. The p-value of less than 0.05 was considered statistically significant.

\section{RESULTS}

\section{MiR-582-5p Was Downregulation in ccRCC Tissues and Cell Lines}

To explore the primary role of miR-582-5p in ccRCC, we quantified the expression of miR-582-5p in $40 \mathrm{ccRCC}$ tissues and their adjacent normal tissues by qRT-PCR. As compared with normal tissues, the expression level of miR-582-5p was significantly decreased in tumor tissues $(P<0.001)$ (Figure 1A). Furthermore, all the patients enrolled in the study was divided into two groups 
according to clinical stage and the status of metastasis respectively. We found that miR-582-5p was high expressed in T1 stage and non-metastatic patients compared with T2-T4 stage and those metastatic patients (Figure 1B). As to cell lines, qRT-PCR results showed that miR-582-5p was significantly decreased in various ccRCC cell lines (including 786-O, 769-P, Caki-1, ACHN and A498) compared with human renal tubular epithelial cells (HK2) (Figure 1C). In conclusion, miR-582-5p was aberrant shrunk in ccRCC especially in higher T stage and metastatic patients.

\section{MiR-582-5p Regulated Cell Proliferation and Invasion}

Considering that miR-582-5p was obviously attenuated in 786-O and Caki-1 cells, these two cell lined were chosen to be transfected with miR-582-5p mimics and corresponding negative control (NC). The transfection efficiency was verified by qRT-PCR (Figure 2A). To investigate the effects of miR-582$5 \mathrm{p}$ on cell proliferation, we first conducted CCK8 assay and found that the viability of cells which transfected with miR-582$5 \mathrm{p}$ mimics significantly decreased after $72 \mathrm{~h}$ compared with $\mathrm{NC}$ (Figure 2B). Furthermore, the results from colony assay reveal that miR-582-5p mimics notably inhibited the colonies (Figure 2C). To uncover the role of miR-582-5p in cell invasion, transwell assay was performed. The results displayed that overexpression of miR-582-5p notably weaken the invasive ability of 786-O and Caki-1 cells (Figure 2D).

\section{MiR-582-5p Promoted Xenograft Tumor Formation}

Furthermore, to determine whether miR-582-5p was involved in tumorigenesis in vivo, xenograft tumor models were established in $\mathrm{BALB} / \mathrm{c}(\mathrm{nu} / \mathrm{nu})$ mice. MiR-582-5p agonists were used to generate a gain-of-function model since miR-582-5p was downregulated in 786-O and Caki-1 cells. The volume of the tumors derived from miR-582-5p overexpressing cells was dramatically reduced compared to that in the tumors derived from control cells (Figure 3A). Additionally, the IHC result of tissue derived from the mice showed that miR-582-5p obviously decrease the expression Ki-67 protein (Figure 3B). In summary, these results demonstrated that miR-582-5p inhibited ccRCC proliferation in vivo.

\section{COL5A1 Was a Potential Target of miR-582-5p}

We performed bioinformatics analysis to predict the target genes of miR-582-5p based on four databases including TargetScan, miRWalk, miRDB and miRanda. As shown in Figure $\mathbf{4 A}, 7$ candidate mRNAs (TBC1D19, COL5A1, NOVA1, CPNE8,

A
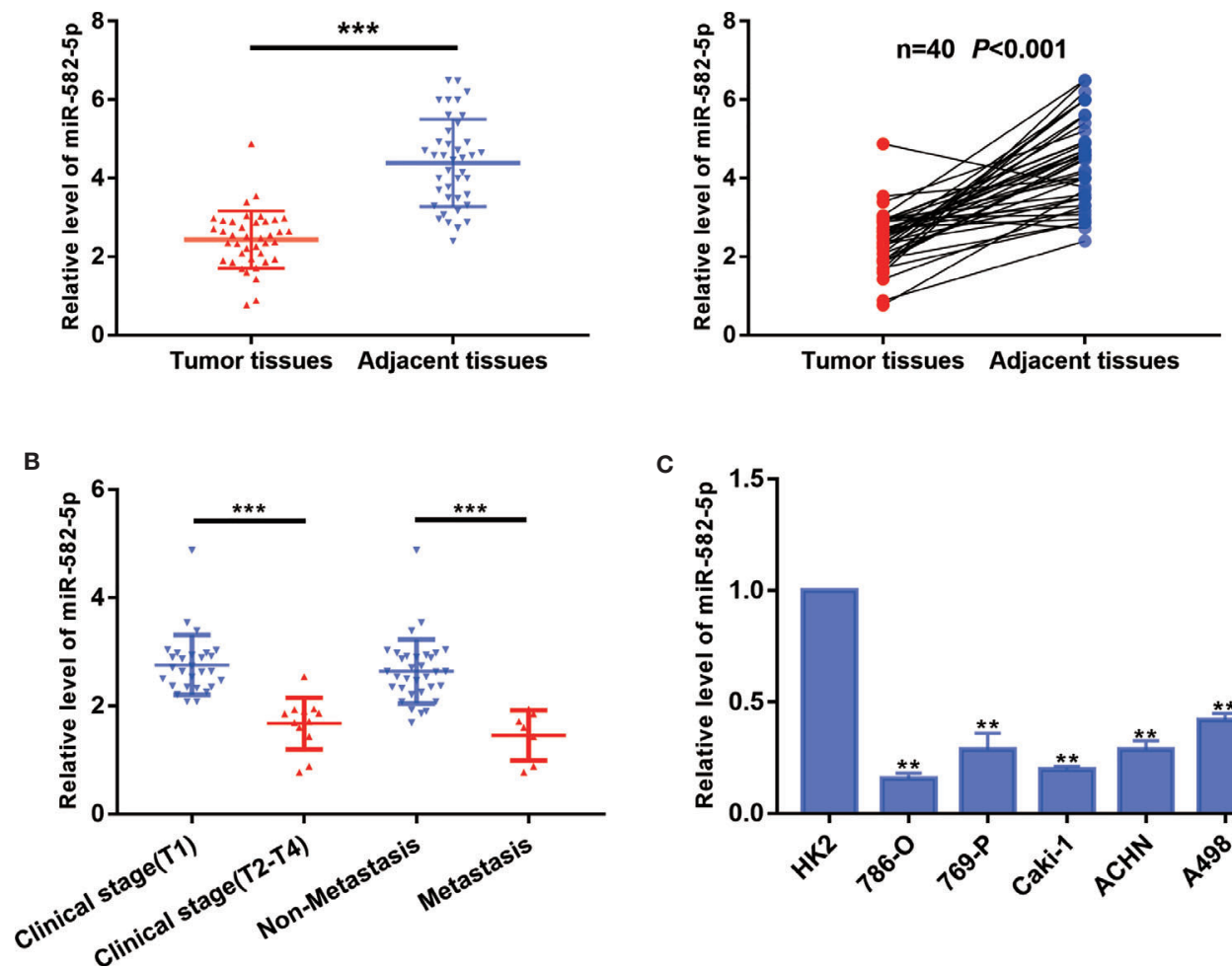

C

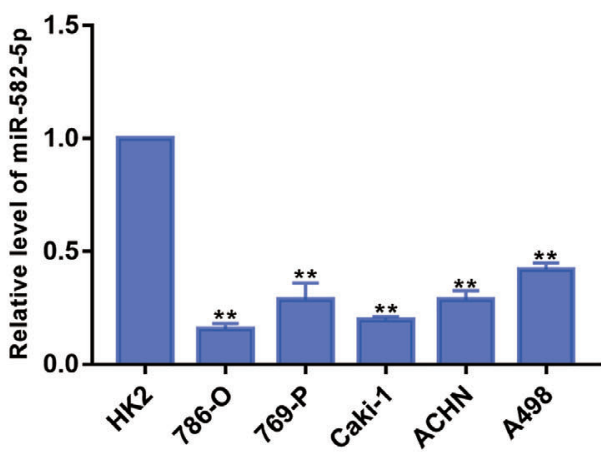

FIGURE 1 | MiR-582-5p was downregulation in ccRCC tissues and cell lines. (A) miR-582-5p level in 40 ccRCC tissues and paired adjacent tissues was investigated by qRT-PCR. (B) Relative expression of miR-582-5p were determined by qRT-PCR in different clinical stage of ccRCC tissues. (C) miR-582-5p level in human normal renal tubular epithelial cell line HK-2 and five ccRCC cell lines was investigated by qRT-PCR; U6 was used as an internal control. The data are shown as the mean $\pm \mathrm{SD}\left({ }^{\star \star} P<0.01 ;{ }^{\star \star \star} P<0.001\right)$. The experiments were repeated three times with similar results. 


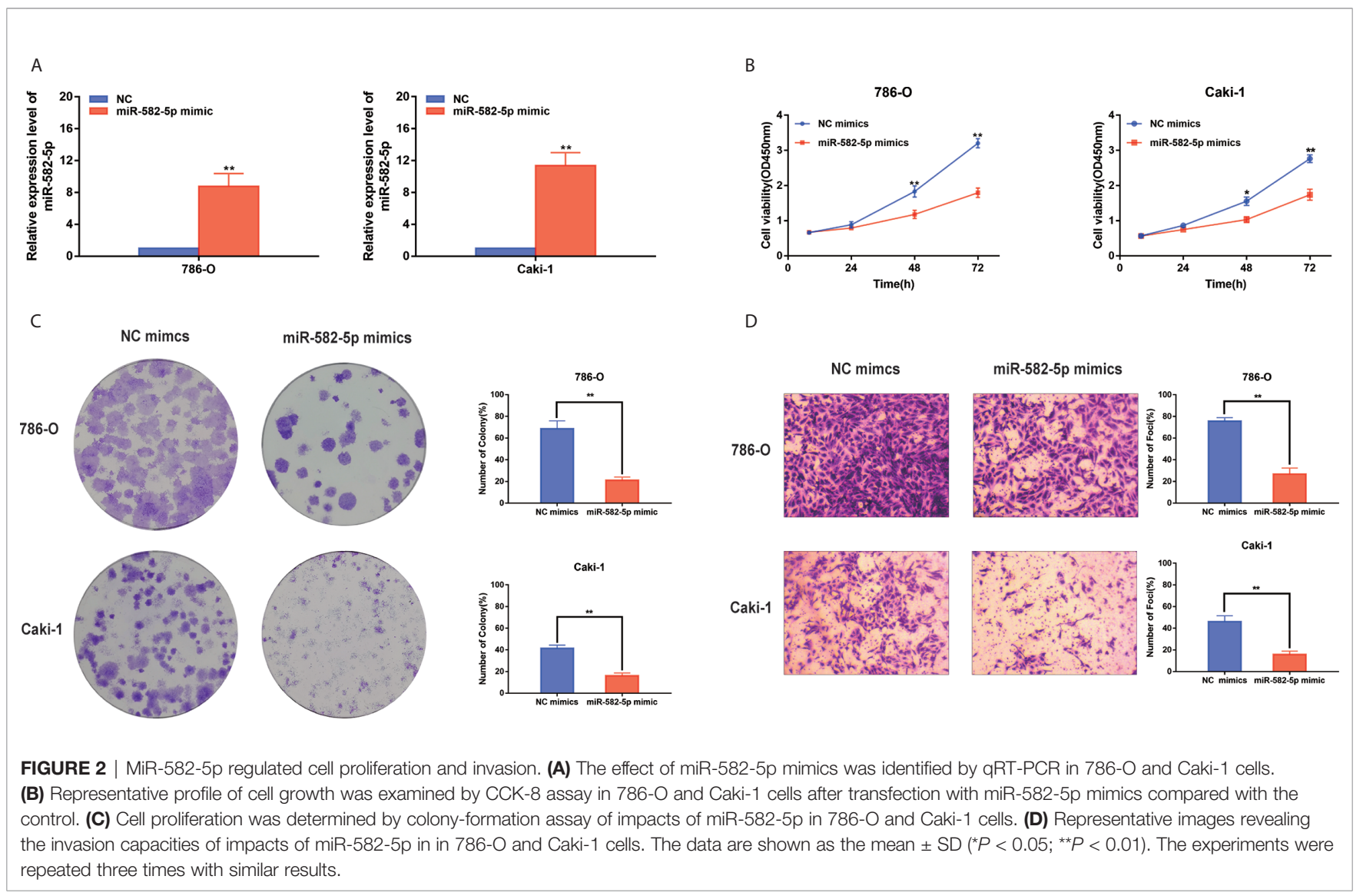

A
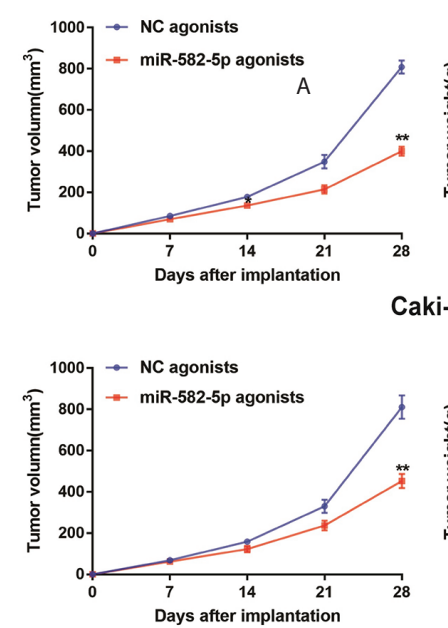

786-0
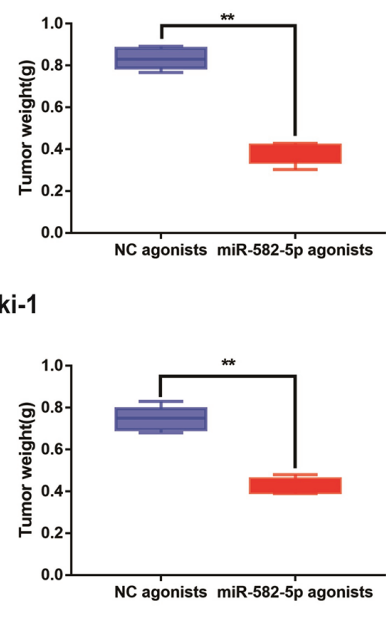

B

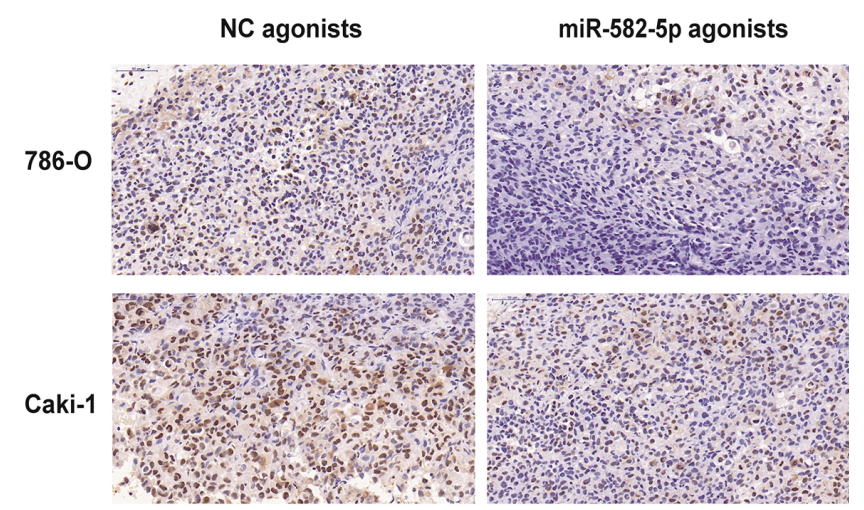

FIGURE 3 | MiR-582-5p promoted xenograft tumor formation. (A) Tumor volume and weight were calculated of the xenograft tumors in subcutaneous xenograft mouse model injected with 786-O and Caki-1 cells transfected with NC agonists and miR-582-5p agonists respectively. (B) The tumor sections from different transfected groups of xenograft mouse models were subjected to immunohistochemistry staining using antibodies against KLF6 (400x). The data are shown as the mean $\pm \mathrm{SD}\left({ }^{\star} P<0.01\right)$. The experiments were repeated three times with similar results. 
MBNL3, MAP3K1 and PAXBP1) were picked. qRT-PCR were used to further validate the expression level of selected mRNAs in 40 paired clinical specimens. Among these mRNAs, 6 genes were significantly aberrant expressed in tumor tissues compared with their adjacent tissues (3 up-regulated and 3 down-regulated) (Figure 4B). Notably, COL5A1 was the most significantly upregulated in the tumor tissues. Further analysis showed that there was a negative correlation between miR-582-5p and COL5A1 in tumor tissues $(r=0.6433, P<0.01)$ (Figure 4C). In addition, we verified the up-regulation expression of COL5A1 in two ccRCC cell lines (786-O and Caki-1) (Figure 4D). To further confirm whether COL5A1 was a direct target of miR-582-5p, bioinformatics analysis was performed to search for the presumed miR-582-5p targeting sites in COL5A1 transcripts. It was found that COL5A1 3'-UTR contained a miR-582-5p-binding site (Figure 4E). In addition,we constructed luciferase reporter vectors containing the wild type (WT) or mutation type (MuT) fragments of COL5A1 3'-UTR. Then the vectors were cotransfected with miR-582-5p mimics or NC mimics into 786-O and Caki-1 cells. Upregulation of miR-582-5p markedly reduced relative luciferase activity in COL5A1-Wt cells, while the relative luciferase activity in the COL5A1-Mut cells showed no significant difference between the transfected miR-582-5p mimics group and the transfected miR-NC group. Taken together, above results revealed that miR-582-5p could influence COL5A1 expression by directly binding to 3'-UTR of COL5A1.

\section{Reconstitution of COL5A1 Partially Rescued the miR-582-5p-Mediated Effects}

The functional relevance of COL5A1 targeting by miR-582-5P was investigated by determining whether COL5A1 overexpression could rescue the inhibitory effects of miR-582-5p on ccRCC cell proliferation and invasion. 786-O and Caki-1 cells were cotransfected with miR-582-5p mimics and pcDNA3.1-COL5A1. Western blot analysis validated the COL5A1 protein in the rescue experiments (Figure 5A). CCK-8 assay and colony formation assay suggested that the overexpression of COL5A1 rescued the inhibitory effect of miR-582-5p on cell proliferation in vitro (Figures 5B, C). Transwell assays showed that the invasion ability inhibited by miR-582-5p was remarkably rescued by the COL5A1 overexpression (Figure 5D). In vivo, the decreased tumor size and weight by miR-582-5p were reversed by COL5A1 overexpression (Figure 5E). These results suggested that miR-582-5p influenced the growth and mobility of ccRCC cells by targeting COL5A1.
A

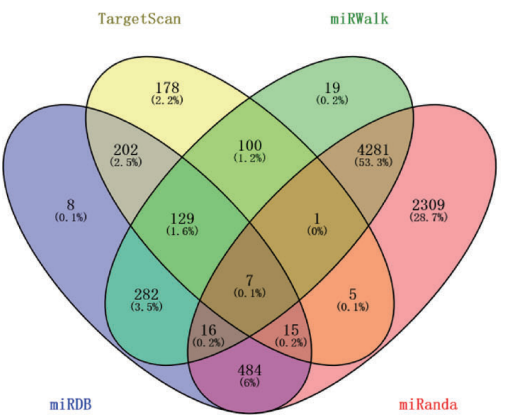

B

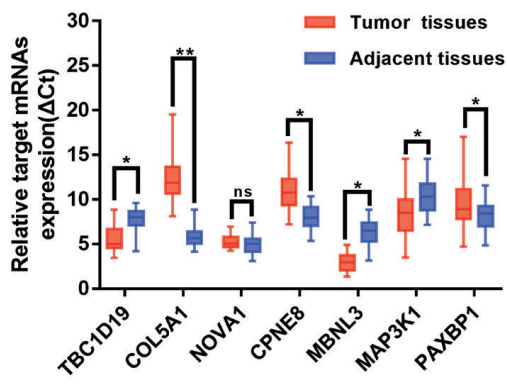

C

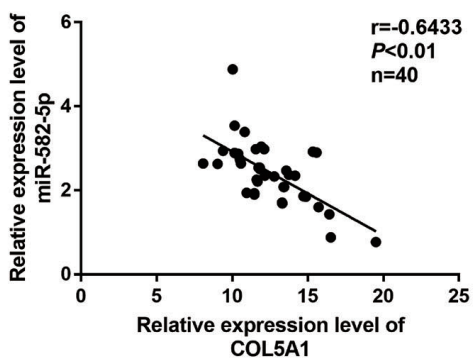

D

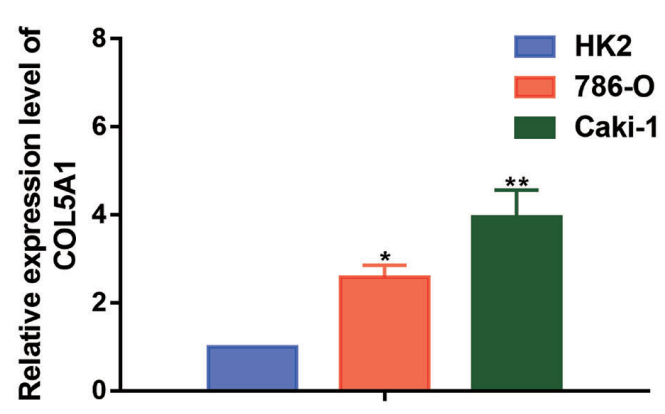

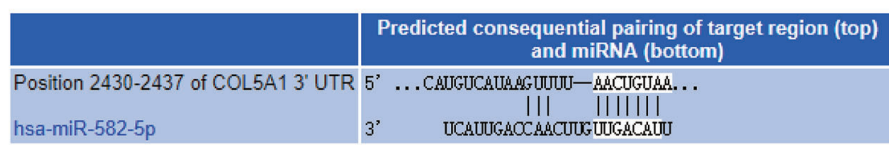

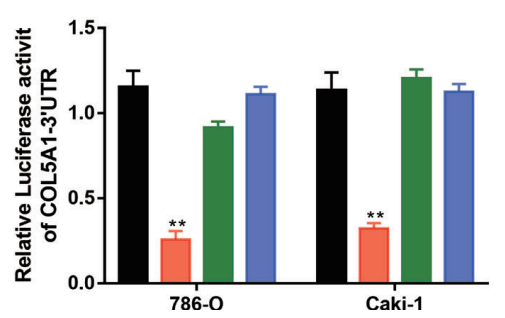

- COL5A1 -Wt+NC mimics

COL5A1 -Wt+miR-582-5p mimics COL5A1 -Mut+NC mimics - COL5A1-Mut+miR-582-5p mimics

FIGURE 4 | COL5A1 was a potential target of miR-582-5p. (A) The candidate gene targets were predicted by intersecting outputs from four distinct prediction algorithms (TargetScan, miRDB, miRWalk, and miRanda). (B) qRT-PCR assay confirmed the relative expression of seven candidate target mRNAs of miR-582-5p in 40 paired ccRCC cancer tissues and corresponding paracancerous normal tissues. (C) There was an inverse correlation between miR-582-5p and COL5A1. (D) COL5A1 mRNA in HK2 cells and ccRCC cell lines (786-O and Caki-1 cells) were investigated by qRT-PCR. (E) The potential miR-582-5p seed region at the 3'UTR of COL5A1 mRNA was computationally predicted. 786-O and Caki-1 cells were co-transfected with miR-582-5p mimics (or NC) with COL5A1-Wt (or COL5A1Mut) vector. Luciferase activity was normalized by the ratio of firefly and Renilla luciferase signals. The data are shown as the mean $\pm \mathrm{SD}\left({ }^{*} P<0.05 ;{ }^{* *} P<0.01\right)$. The experiments were repeated three times with similar results. 
A

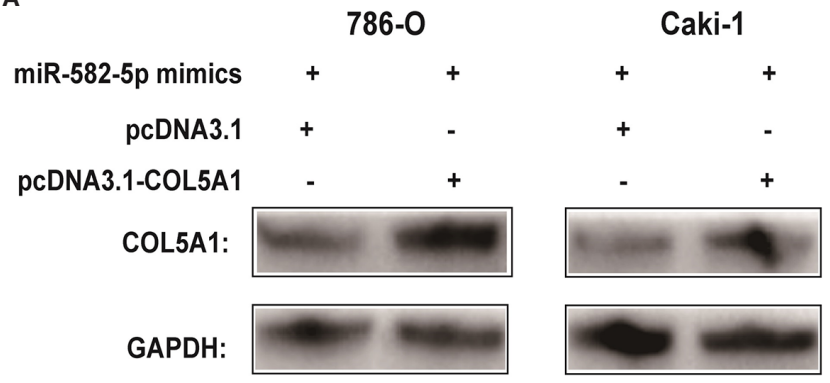

C
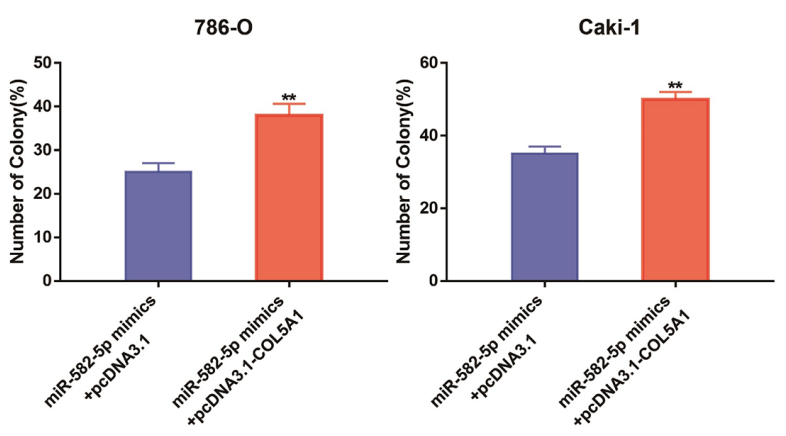

E

786-0
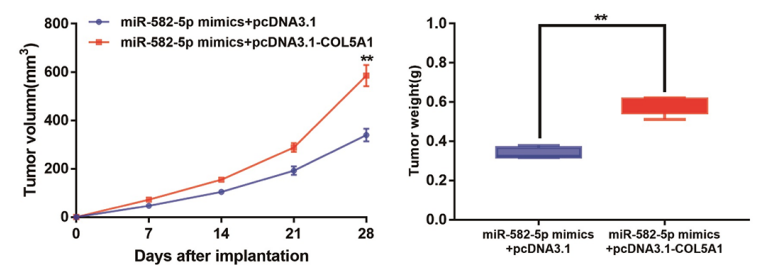

B

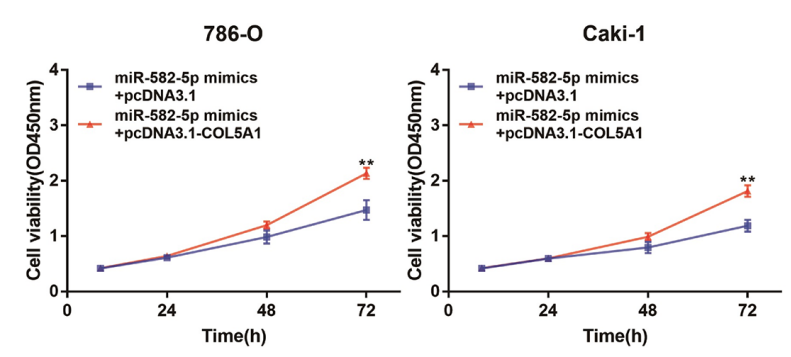

D

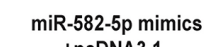
+pcDNA3.1
-582-5p mimics

miR-582-5p mimics +pcDNA3.1-COL5A1

$786-0$
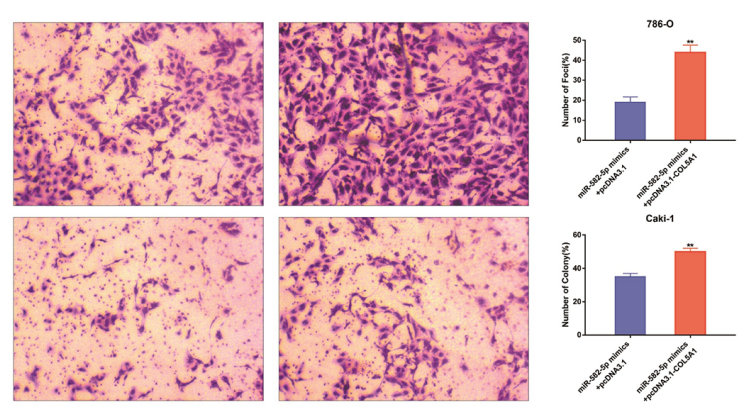

Caki-1
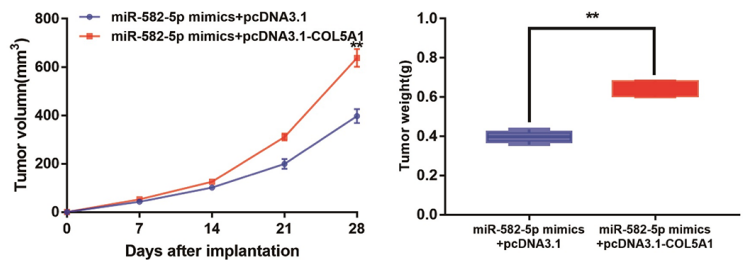

FIGURE 5 | Reconstitution of COL5A1 partially rescued the miR-582-5p-mediated effects. (A) Expression levels of COL5A1 in 786-O and Caki-1 cells which cotransfected with miR-582-5p mimics and pcDNA3.1-COL5A1 (or pcDNA3.1) were detected by western blot. GAPDH was used as an internal control. (B) CCK-8 assay of 786-O and Caki-1 cells in different transfected groups. (C) Cell proliferation was determined by colony-formation assay of different transfected groups in 786-O and Caki-1 cells. (D) Representative images revealing the invasion capacities of impacts of different transfected groups in 786-O and Caki-1 cells. (E) Tumor volume and weight were calculated of the xenograft tumors in subcutaneous xenograft mouse model injected with 786-O and Caki-1 cells co-transfected with COL5A1 and miR-582-5p mimics or co-transfected with vector and miR-582-5p mimics respectively. The data are shown as the mean \pm SD (** $P<0.01)$. The experiments were repeated three times with similar results.

\section{RUNX1 Was an Upstream Regulator of miR-582-5p}

To identify upstream regulators that may be responsible for miR582-5p decrease in ccRCC, based on TransmiR database, we found that numerous genes might be potential upstream regulator of miR-582-5p (Figure 6A). Of them, the putative score of RUNX1 binding to miR-582-5p was the highest (Figure 6B). Furthermore, ChIP assay indicated that RUNX1 binds to the putative binding site upstream of miR-582-5p (Figure 6C). The efficiency of overexpression of RUNX1 was identified by qRT-PCR (Figure 6D). Overexpression of RUNX1 led to decreased miR-582-5p and increased COL5A1 expression which indicated that there was an axis among RUNX1/miR-582-5p/COL5A1 signaling (Figures 6E, F). According to previous research, we already known that
RUNX1 was over-expression in human ccRCC, and high protein expression correlates with poorer survival. Additionally, deletion of RUNXL1 was able to disrupt tumor cell growth both in vitro and in vivo (24). Subsequently, we recruited a rescue assay to figure out whether miR-582-5p could rescue the overexpressed RUNX1 effect. As shown in Figure 6G, after co-transfection of miR-582-5p mimics with pcDNA 3.1-RUNX1 or vector, the expression level of COL5A1 were detected. Moreover, the results showed that miR-582-5p overexpression could partially rescue the effects of RUNX1 on cell proliferation and invasion in ccRCC cell lines (Figures 6H-J). In vivo, the miR-582-5p induced tumor shrinkage was reversed by RUNX1 overexpression (Figure 6K). Overall, the total data indicated that RUNX1 contributed to the tumor growth by modulating miR-582-5p transcription. 
A

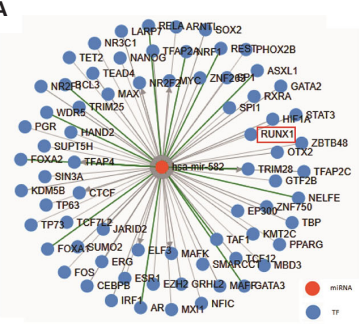

D

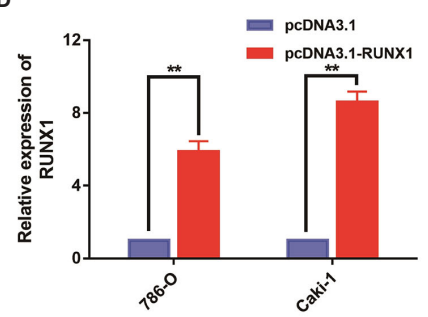

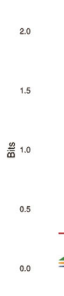

E

\section{Putative binding site: aattgtggtta}

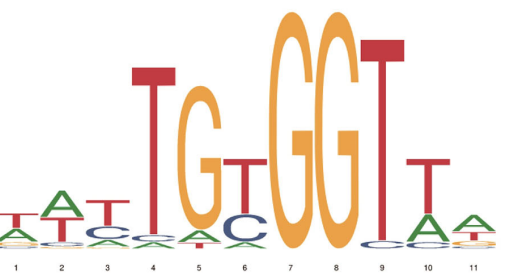

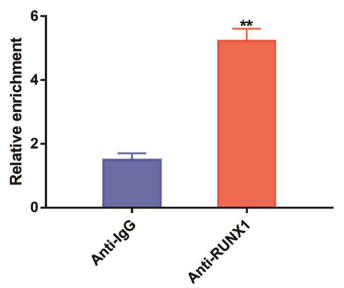

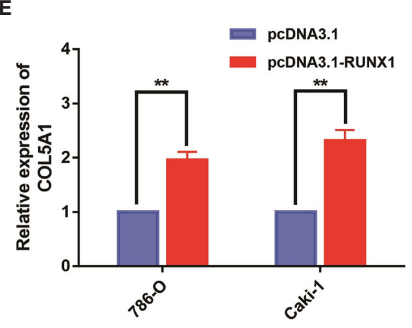

F

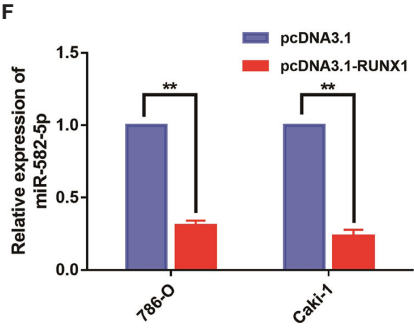

G
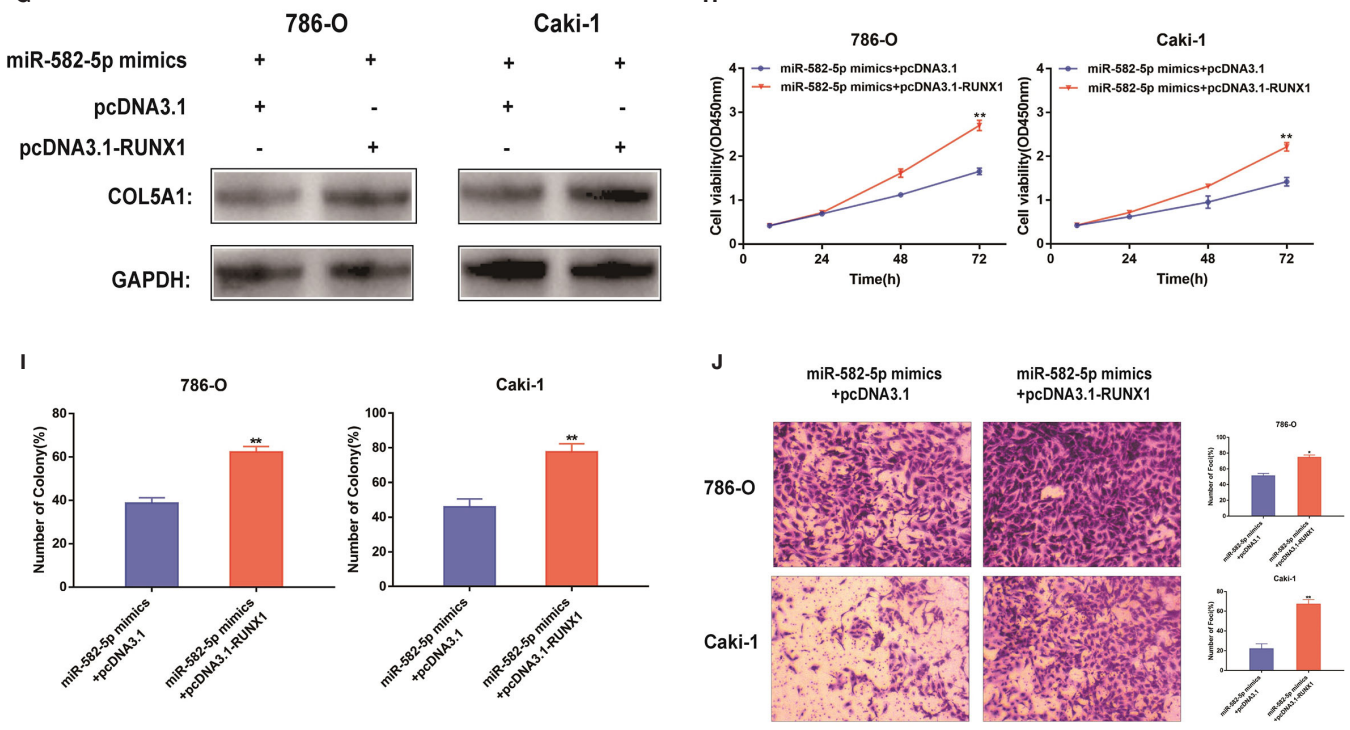

K

786-0
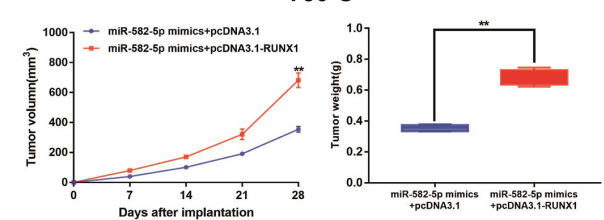

Caki-1

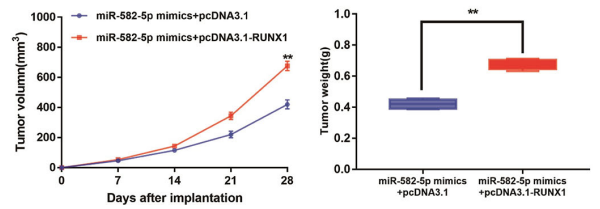

FIGURE 6 | RUNX1 was an upstream regulator of miR-582-5p. (A) The candidate upstream regulators were predicted by TransmiR database. (B) Based on TransmiR database, the putative score of RUNX1 binding to miR-582-5p was the highest. (C) ChIP assay indicated the RUNX1 binds to the putative binding site upstream of miR-582-5p. (D) The effect of overexpression RUNX1 by pcDNA3.1 was identified by qRT-PCR. (E, F) Levels of miR-582-5p and COL5A1 responding overexpression RUNX1 were detected by qRT-PCR. (G) Expression levels of COL5A1 in 786-O and Caki-1 cells which co-trnsfected with miR-582-5p mimics and pcDNA3.1-RUNX1 (or pcDNA3.1) were detected by western blot. GAPDH was used as an internal control. (H, I) CCK-8 and colony-formation assays were the influence of 786-O and Caki-1 cells which co-transfected with miR-582-5p mimics and pcDNA3.1-RUNX1 (or pcDNA3.1) on cell proliferation respectively. (J) Cell invasion was determined after co-transfected with miR-582-5p mimics and pcDNA3.1-RUNX1 or pcDNA3.1. (K) Tumor volume and weight were calculated of the xenograft tumors in subcutaneous xenograft mouse model injected with 786-O and Caki-1 cells co-transfected with RUNX1 and miR-582-5p mimics or cotransfected with vector and miR-582-5p mimics respectively. The data are shown as the mean $\pm \mathrm{SD}\left({ }^{\star} P<0.05 ;{ }^{\star \star} P<0.01\right)$. The experiments were repeated three times with similar results. 


\section{DISCUSSION}

RCC is a heterogeneous group of epithelial tumors, among which clear cell RCC (ccRCC) is the most common and accounts for 70$80 \%$ of the reported cases of RCC (25). Lack of effective diagnostics in the early stages of the disease, increasing mortality rate, and resistance to therapies in patients with metastatic ccRCC emphasize the need to discover new biomarkers that are applicable for the early diagnosis of ccRCC and detection of metastasis. To this end, many studies have been conduct with the aim of generating molecular markers that regulate RCC progression and metastasis. To our knowledge, many non-coding RNAs including miRNAs play critical role of either oncogene or suppressor in RCC (26). In this study, we discovered a new molecular mechanism related to the proliferation and invasion of ccRCC. As shown in the schematic diagram (Figure 7), RUNX1-mediated miR-582-5p regulates the cell proliferation and invasion of ccRCC by targeting COL5A1.
Our study first proved the expression pattern and mechanism of miR-582-5p in ccRCC. Briefly, miR-582-5pexpression was obviously decreased in ccRCC tumor tissues and cells. Moreover, low miR-582$5 \mathrm{p}$ expression was related to higher $\mathrm{T}$ stage and the status of metastasis in ccRCC patients. Findings also revealed that miR-582$5 \mathrm{p}$ overexpression prominently inhibited RCC cell proliferation and invasion capacities. Additionally, cell line-derived xenograft model was utilized which further confirmed the tumor-suppressive role of miR-582-5p in ccRCC. The result was in accordance with previous findings in glioblastoma, prostate cancer, gastric cancer, none-small cell lung cancer, bladder cancer, etc. $(13,14,16,21,22)$.

As we all know, miRNAs normally regulate biological process by binding to downstream mRNA. Therefore, the identification of target genes of miRNAs plays an important role in the diagnosis and treatment of tumors (9-12). Based on bioinformatic analysis, we found 7 candidate genes among which COL5A1 was the most significant upregulated gene. Collagen type V (COL5) is a

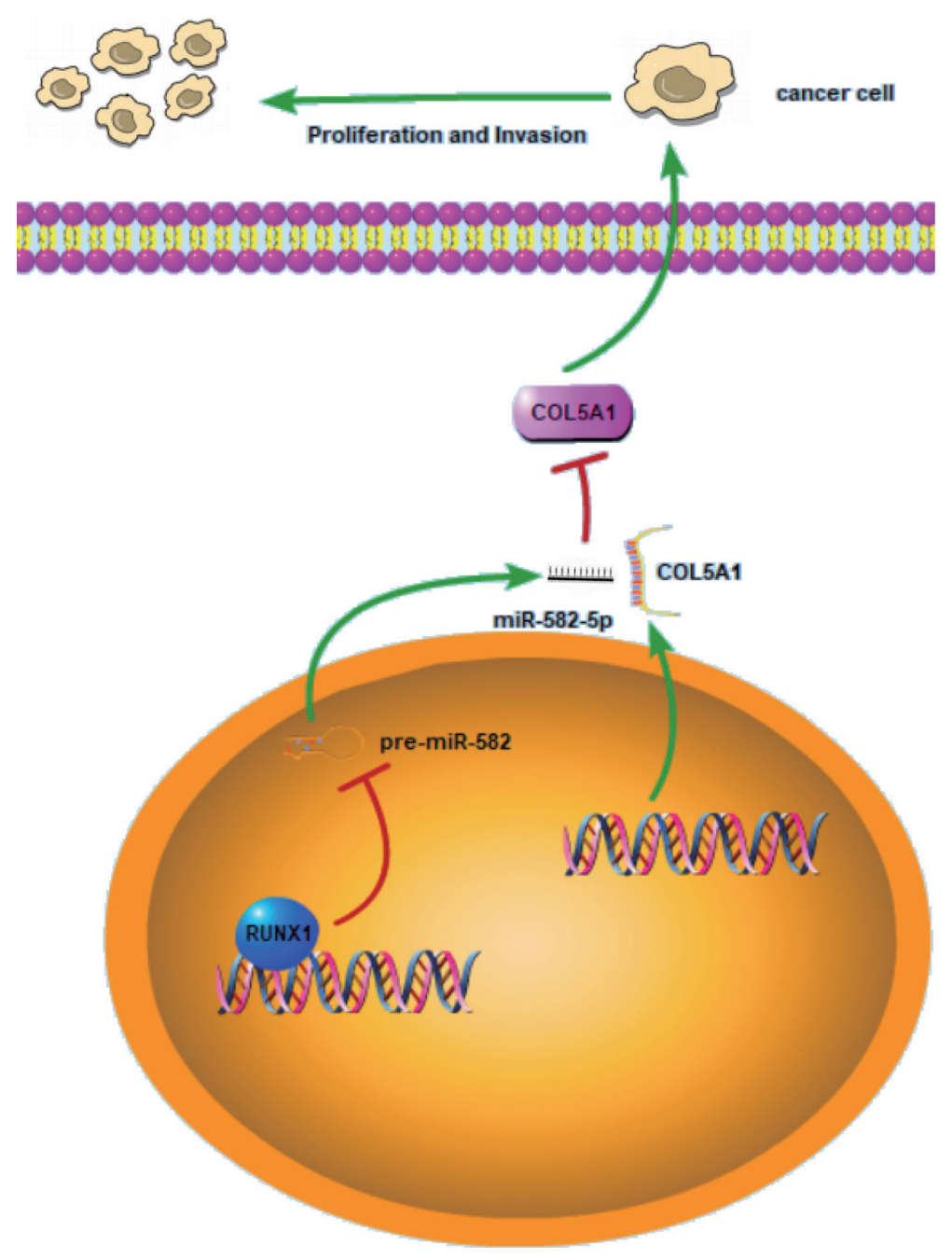

FIGURE 7 | Schematic diagram for miR-582-5p, mediated by RUNX1, regulates cell proliferation and invasion by targeting COL5A1 in ccRCC. RUNX1 was verified to bind to miR-582-5p promoter and attenuated miR-582-5p expression in ccRCC. miR-582-5p regulated cell proliferation and invasion in ccRCC by targeting COL5A1. The experiments were repeated three times with similar results. 
regulatory fibril-forming collagen which has at least three different molecular isoforms-COL5A1,COL5A2,COL5A3 (27). Previous evidences show that COL5A1, a member of collagen family protein, was related to extracellular matrix (ECM) which is known as an essential event in metastasis of RCC $(28,29)$. In fact, COL5A1 is a core gene in metastatic RCC whose expression level correlates with poor survival $(30,31)$. Previous study by Feng $\mathrm{G}$ et al. has found COL5A1 promoted ccRCC cell proliferation, apoptosis, migration, invasion in vitro (32). Therefore we presumed that COL5A1 was the target of miR-582-5p. To further confirm this, we constructed a wild-type plasmid and a mutant plasmid (COL5A1-Wt or COL5A1Mut) of the luciferase reporter vector, and the results showed that luciferase activity in COL5A1-Wt transfected miR-582-5p mimics was significantly lower than the COL5A1-Wt luciferase activity in the transfected miR-NC group. In addition, it was found no significant difference in the luciferase-Mut activity between the transfected miR582-5p mimics group and the luciferase-Mut activity in the transfected miR-NC group, suggesting that miR-582-5p could inhibit COL5A1 expression. In addition, we then performed rescue assay to confirm the relation between miR-582-5p and COL5A1. The results showed that overexpression of COL5A1 could partially reverse the inhibition of cell proliferation and invasion by miR-582-5p.

In addition, the upstream regulators such as transcription factors may be responsible for dysregulated expression of miRNAs in cancers. By using TransmiR database, we identified RUNX1 as an upstream regulator of miR-582-5p expression. Runt-related transcription factor 1 (RUNX1) is one subunit of the core-binding factors (CBFs) that binds to the core element of many enhancers and promoters (33). RUNX1 was involved in the development of normal hematopoiesis, while its mutation and translocations had been associated with several types of leukemia $(34,35)$. RUNX1, as an oncogene and anti-oncogene, has also received increasing attention in many solid tumors including breast cancer, gastric cancer and colorectal cancer (36-38). Recent studies have shown that RUNX1 participated into many biological processes by regulating downstream pathway. RUNX1 promoted renal tubular Epithelial-to-Mesenchymal Transition (EMT) and kidney fibrosis by regulating TGF- $\beta$ (39). In addition, another study has found that RUNX1 suppressed the expression of the miR-17 92 cluster in ovarian cancer (40). Thus, RUNX1 could regulate expression of both protein-coding genes and noncoding genes such as miRNAs in several biological processes. In our study, ChIP assay indicated the RUNX1 bind to the binding site upstream of miR-582-5p. Over-expression of RUNX1 led to decreased miR-582-5p expression and increased COL5A1 expression, which demonstrates that there is an axis among RUNX1/miR-582-5p/COL5A1 signaling. Over-expression of RUNX1 caused increasing cell proliferation and invasion,

\section{REFERENCES}

1. Bray F, Ferlay J, Soerjomataram I, Siegel RL, Torre LA, Jemal A. Global cancer statistics 2018: GLOBOCAN estimates of incidence and mortality worldwide for 36 cancers in 185 countries. CA Cancer J Clin (2018) 68(6):394-424. doi: $10.3322 /$ caac. 21492

2. Moch H, Cubilla AL, Humphrey PA, Reuter VE, Ulbright TM. The 2016 WHO Classification of Tumours of the Urinary System and Male Genital suggesting that RUNX1 contributed to the tumor growth by modulating miR-582-5p expression.

\section{CONCLUSION}

In summary, the study availed a better understanding of the function of miR-582-5p in ccRCC. We confirmed a new antionco-miRNA, miR-582-5p, mediated by RUNX1, regulated cell proliferation and invasion by targeting COL5A1 in ccRCC. These findings indicated a tumor suppressor role of miR-582-5p in ccRCC development and might serve as a potential therapeutic target in ccRCC.

\section{DATA AVAILABILITY STATEMENT}

The raw data supporting the conclusions of this article will be made available by the authors without undue reservation.

\section{ETHICS STATEMENT}

The studies involving human participants were reviewed and approved by the ethics committee of First Affiliated Hospital of Nanjing Medical University. The patients/participants provided their written informed consent to participate in this study. The animal study was reviewed and approved by the animal ethics committee of Nanjing Medical University. Written informed consent was obtained from the individual(s) for the publication of any potentially identifiable images or data included in this article.

\section{AUTHOR CONTRIBUTIONS}

JX and ZW: study design and funding support; JX, SZ and FQ: celluar experimnets; SZ and KZ: animal experiments; JX and PC: Data collection and analyses; JX, SZ and KZ: manuscript writing and editing; JY and ZW: supervision. All authors contributed to the work and approved it for publication.

\section{ACKNOWLEDGMENTS}

This work was supported by National Natural Science Foundation of China (grant no. 81800597 and 81771640). 
5. Capitanio U, Bensalah K, Bex A, Boorjian SA, Bray F, Coleman J, et al. Epidemiology of Renal Cell Carcinoma. Eur Urol (2019) 75(1):74-84. doi: 10.1016/j.eururo.2018.08.036

6. Campbell L, Jasani B, Griffiths DF, Gumbleton M. Phospho-4e-BP1 and eIF4E overexpression synergistically drives disease progression in clinically confined clear cell renal cell carcinoma. Am J Cancer Res (2015) 5(9):2838-48.

7. Bartel DP. MicroRNAs: target recognition and regulatory functions. Cell (2009) 136(2):215-33. doi: 10.1016/j.cell.2009.01.002

8. Baltimore D, Boldin MP, O'Connell RM, Rao DS, Taganov KD. MicroRNAs: new regulators of immune cell development and function. Nat Immunol (2008) 9(8):839-45. doi: 10.1038/ni.f.209

9. Calin GA, Croce CM. MicroRNA signatures in human cancers. Nat Rev Cancer (2006) 6(11):857-66. doi: 10.1038/nrc1997

10. Bushati N, Cohen SM. microRNA functions. Annu Rev Cell Dev Biol (2007) 23:175-205. doi: 10.1146/annurev.cellbio.23.090506.123406

11. Esquela-Kerscher A, Slack FJ. Oncomirs - microRNAs with a role in cancer. Nat Rev Cancer (2006) 6(4):259-69. doi: 10.1038/nrc1840

12. Dalmay T, Edwards DR. MicroRNAs and the hallmarks of cancer. Oncogene (2006) 25(46):6170-5. doi: 10.1038/sj.onc.1209911

13. Liu J, Liu S, Deng X, Rao J, Huang K, Xu G, et al. MicroRNA-582-5p suppresses non-small cell lung cancer cells growth and invasion via downregulating NOTCH1. PLoS One (2019) 14(6):e0217652. doi: 10.1371/ journal.pone.0217652

14. Huang S, Zou C, Tang Y, Wa Q, Peng X, Chen X, et al. miR-582-3p and miR-582$5 p$ Suppress Prostate Cancer Metastasis to Bone by Repressing TGF-beta Signaling. Mol Ther Nucleic Acids (2019) 16:91-104. doi: 10.1016/j.omtn.2019.01.004

15. Wang WW, Chen B, Lei CB, Liu GX, Wang YG, Yi C, et al. miR-582-5p inhibits invasion and migration of salivary adenoid cystic carcinoma cells by targeting FOXC1. Japanese J Clin Oncol (2017) 47(8):690-8. doi: 10.1093/jjco/ hyx073

16. Jin Y, Tao LP, Yao SC, Huang QK, Chen ZF, Sun YJ, et al. MicroRNA-582-5p suppressed gastric cancer cell proliferation via targeting AKT3. Eur Rev Med Pharmacol Sci (2017) 21(22):5112-20. doi: 10.26355/eurrev_201711_13827

17. Shu Z, Chen L, Ding D. miR-582-5P induces colorectal cancer cell proliferation by targeting adenomatous polyposis coli. World J Surg Oncol (2016) 14(1):239. doi: 10.1186/s12957-016-0984-4

18. Zhang Y, Huang W, Ran Y, Xiong Y, Zhong Z, Fan X, et al. miR-582-5p inhibits proliferation of hepatocellular carcinoma by targeting CDK1 and AKT3. Tumour Biol J Int Soc Oncodevelopmental Biol Med (2015) 36 (11):8309-16. doi: 10.1007/s13277-015-3582-0

19. Zhang X, Zhang Y, Yang J, Li S, Chen J. Upregulation of miR-582-5p inhibits cell proliferation, cell cycle progression and invasion by targeting Rab27a in human colorectal carcinoma. Cancer Gene Ther (2015) 22(10):475-80. doi: $10.1038 /$ cgt.2015.44

20. Maeno A, Terada N, Uegaki M, Goto T, Okada Y, Kobayashi T, et al. Upregulation of miR-582-5p regulates cellular proliferation of prostate cancer cells under androgen-deprived conditions. Prostate (2014) 74(16):1604-12. doi: $10.1002 /$ pros.22877

21. Floyd DH, Zhang Y, Dey BK, Kefas B, Breit H, Marks K, et al. Novel antiapoptotic microRNAs 582-5p and 363 promote human glioblastoma stem cell survival via direct inhibition of caspase 3, caspase 9, and Bim. PloS One (2014) 9(5):e96239. doi: 10.1371/journal.pone.0096239

22. Uchino K, Takeshita F, Takahashi RU, Kosaka N, Fujiwara K, Naruoka H, et al. Therapeutic effects of microRNA-582-5p and $-3 p$ on the inhibition of bladder cancer progression. Mol Ther J Am Soc Gene Ther (2013) 21(3):610-9. doi: $10.1038 / \mathrm{mt} .2012 .269$

23. Lu YC, Chen YJ, Wang HM, Tsai CY, Chen WH, Huang YC, et al. Oncogenic function and early detection potential of miRNA-10b in oral cancer as identified by microRNA profiling. Cancer Prev Res (Philadelphia Pa) (2012) 5(4):665-74. doi: 10.1158/1940-6207.capr-11-0358

24. Rooney N, Mason SM, McDonald L, Dabritz JHM, Campbell KJ, Hedley A, et al. RUNX1 Is a Driver of Renal Cell Carcinoma Correlating with Clinical Outcome. Cancer Res (2020) 80(11):2325-39. doi: 10.1158/0008-5472.CAN19-3870

25. Cairns P. Renal cell carcinoma. Cancer Biomark (2010) 9(1-6):461-73. doi: 10.3233/CBM-2011-0176
26. Braga EA, Fridman MV, Loginov VI, Dmitriev AA, Morozov SG. Molecular Mechanisms in Clear Cell Renal Cell Carcinoma: Role of miRNAs and Hypermethylated miRNA Genes in Crucial Oncogenic Pathways and Processes. Front Genet (2019) 10:320. doi: 10.3389/fgene.2019.00320

27. Mak KM, Png CY, Lee DJ. Type V Collagen in Health, Disease, and Fibrosis. Anat Rec (Hoboken) (2016) 299(5):613-29. doi: 10.1002/ar.23330

28. Mikami S, Oya M, Mizuno R, Kosaka T, Katsube K, Okada Y. Invasion and metastasis of renal cell carcinoma. Med Mol Morphol (2014) 47(2):63-7. doi: 10.1007/s00795-013-0064-6

29. Ho TH, Serie DJ, Parasramka M, Cheville JC, Bot BM, Tan W, et al. Differential gene expression profiling of matched primary renal cell carcinoma and metastases reveals upregulation of extracellular matrix genes. Ann Oncol (2017) 28(3):604-10. doi: 10.1093/annonc/mdw652

30. Li C, Shao T, Bao G, Gao Z, Zhang Y, Ding H, et al. Identification of potential core genes in metastatic renal cell carcinoma using bioinformatics analysis. Am J Transl Res (2019) 11(11):6812-25.

31. Boguslawska J, Kedzierska H, Poplawski P, Rybicka B, Tanski Z, PiekielkoWitkowska A. Expression of Genes Involved in Cellular Adhesion and Extracellular Matrix Remodeling Correlates with Poor Survival of Patients with Renal Cancer. J Urol (2016) 195(6):1892-902. doi: 10.1016/ j.juro.2015.11.050

32. Feng G, Ma HM, Huang HB, Li YW, Zhang P, Huang JJ, et al. Overexpression of COL5A1 promotes tumor progression and metastasis and correlates with poor survival of patients with clear cell renal cell carcinoma. Cancer Manag Res (2019) 11:1263-74. doi: 10.2147/CMAR.S188216

33. Okuda T, van Deursen J, Hiebert SW, Grosveld G, Downing JR. AML1, the target of multiple chromosomal translocations in human leukemia, is essential for normal fetal liver hematopoiesis. Cell (1996) 84(2):321-30. doi: 10.1016/ s0092-8674(00)80986-1

34. Sood R, Kamikubo Y, Liu P. Role of RUNX1 in hematological malignancies. Blood (2017) 129(15):2070-82. doi: 10.1182/blood-2016-10-687830

35. Speck NA, Gilliland DG. Core-binding factors in haematopoiesis and leukaemia. Nat Rev Cancer (2002) 2(7):502-13. doi: 10.1038/nrc840

36. Han R, Wei J, Zhang H, Su X, Chu X, Chen Y, et al. Influence of TS (rs34743033) and RUNX1 (rs2014300) gene polymorphisms on survival outcomes of fluorouracil-based chemotherapy in Chinese advanced gastric cancer patients. Cancer Manag Res (2018) 10:1429-37. doi: 10.2147/ CMAR.S158647

37. Hong D, Fritz AJ, Finstad KH, Fitzgerald MP, Weinheimer A, Viens AL, et al. Suppression of Breast Cancer Stem Cells and Tumor Growth by the RUNX1 Transcription Factor. Mol Cancer Res (2018) 16(12):1952-64. doi: 10.1158/ 1541-7786.MCR-18-0135

38. Li Q, Lai Q, He C, Fang Y, Yan Q, Zhang Y, et al. RUNX1 promotes tumour metastasis by activating the Wnt/beta-catenin signalling pathway and EMT in colorectal cancer. J Exp Clin Cancer Res (2019) 38(1):334. doi: 10.1186/ s13046-019-1330-9

39. Zhou T, Luo M, Cai W, Zhou S, Feng D, Xu C, et al. Runt-Related Transcription Factor 1 (RUNX1) Promotes TGF-beta-Induced Renal Tubular Epithelial-to-Mesenchymal Transition (EMT) and Renal Fibrosis through the PI3K Subunit p110delta. EBioMedicine (2018) 31:217-25. doi: 10.1016/j.ebiom.2018.04.023

40. Xiao L, Peng Z, Zhu A, Xue R, Lu R, Mi J, et al. Inhibition of RUNX1 promotes cisplatin-induced apoptosis in ovarian cancer cells. Biochem Pharmacol (2020) 180:114116. doi: 10.1016/j.bcp.2020.114116

Conflict of Interest: The authors declare that the research was conducted in the absence of any commercial or financial relationships that could be construed as a potential conflict of interest.

Copyright $\odot 2021$ Xue, Zhu, Qi, Zhu, Cao, Yang and Wang. This is an open-access article distributed under the terms of the Creative Commons Attribution License (CC BY). The use, distribution or reproduction in other forums is permitted, provided the original author(s) and the copyright owner(s) are credited and that the original publication in this journal is cited, in accordance with accepted academic practice. No use, distribution or reproduction is permitted which does not comply with these terms. 